\title{
Chitosan-Based Macromolecular Biomaterials for the Regeneration of Chondroskeletal and Nerve Tissue
}

\author{
Giulio D. Guerra, ${ }^{1}$ Niccoletta Barbani, ${ }^{2}$ Mariacristina Gagliardi, ${ }^{2}$ \\ Elisabetta Rosellini, ${ }^{2}$ and Caterina Cristallini ${ }^{1}$ \\ ${ }^{1}$ Institute for Composite and Biomedical Materials (IMCB), Research Unit of Pisa, Italian National Research Council (CNR), \\ Largo Lucio Lazzarino, 56122 Pisa, Italy \\ ${ }^{2}$ Department of Chemical Engineering, Industrial Chemistry and Materials Science (DICCISM), University of Pisa, \\ Largo Lucio Lazzarino, 56122 Pisa, Italy
}

Correspondence should be addressed to Giulio D. Guerra, guerra@diccism.unipi.it

Received 1 February 2011; Revised 26 April 2011; Accepted 21 June 2011

Academic Editor: Alejandro Sosnik

Copyright ( $) 2011$ Giulio D. Guerra et al. This is an open access article distributed under the Creative Commons Attribution License, which permits unrestricted use, distribution, and reproduction in any medium, provided the original work is properly cited.

\begin{abstract}
The use of materials, containing the biocompatible and bioresorbable biopolymer poly $(1 \rightarrow 4)$-2-amino-2-deoxy- $\beta$-D-glucan, containing some $\mathrm{N}$-acetyl-glucosamine units (chitosan, $\mathrm{CHI}$ ) and/or its derivatives, to fabricate devices for the regeneration of bone, cartilage and nerve tissue, was reviewed. The CHI-containing devices, to be used for bone and cartilage regeneration and healing, were tested mainly for in vitro cell adhesion and proliferation and for insertion into animals; only the use of $\mathrm{CHI}$ in dental surgery has reached the clinical application. Regarding the nerve tissue, only a surgical repair of a $35 \mathrm{~mm}$-long nerve defect in the median nerve of the right arm at elbow level with an artificial nerve graft, comprising an outer microporous conduit of CHI and internal oriented filaments of poly(glycolic acid), was reported. As a consequence, although many positive results have been obtained, much work must still be made, especially for the passage from the experimentation of the CHI-based devices, in vitro and in animals, to their clinical application.
\end{abstract}

\section{Introduction}

Chitosan (CHI) is a $\operatorname{poly}(1 \rightarrow 4)$-2-amino-2-deoxy- $\beta$ - $\mathrm{D}$ glucan, containing some $\mathrm{N}$-acetyl-glucosamine units (Figure 1), obtained by partial deacetylation of chitin, the main component of the exoskeleton of crustaceans, and it is generally considered as biocompatible and biodegradable $[1,2]$; chitin and $\mathrm{CHI}$ are the most abundant polysaccharides among those containing amino sugars [3]. CHI was used some years ago, by the authors' group, as a template for the polymerization of acrylic acid and sodium 4-sty-renesulfonate [4]; the polyelectrolyte complex obtained with the first monomer showed a good cytocompatibility, while that with the second one seemed to influence negatively the cell proliferation [5]. Very many studies have been done on CHI and its derivatives as materials for the fabrication of scaffolds, used for tissue engineering and regeneration. The early studies about the possibility of using $\mathrm{CHI}$ and its derivatives in the food and biomedical sciences sand industries regarded mainly the immobilization of enzymes on the polysaccharide $[6,7]$. The results obtained in this field up to 1980 were the argument of a review by A. Muzzarelli [3]. Another interesting argument of these early studies regarded the chelating ability of CHI towards metal cations [8-10], which was found later to facilitate the tissue mineralization in tooth implantation [11]; furthermore, it was proposed more recently that the CHI-metal interaction modes might be involved in the controlled bioactivity of CHI [12]. In 2005, R. A. A. Muzzarelli and C. Muzzarelli thoroughly reviewed the researches made on $\mathrm{CHI}$ and its derivatives as biomaterials [13]. In 2008, Korean biotechnologists reviewed the use of $\mathrm{CHI}$ and $\mathrm{CHI}$ derivatives for tissue engineering of various organs, between which there were bone, cartilage, and nerves [14]. This paper will summarize mainly the current body of growing literature, where a use of $\mathrm{CHI}$ for cartilage, bone, and nerve tissue 


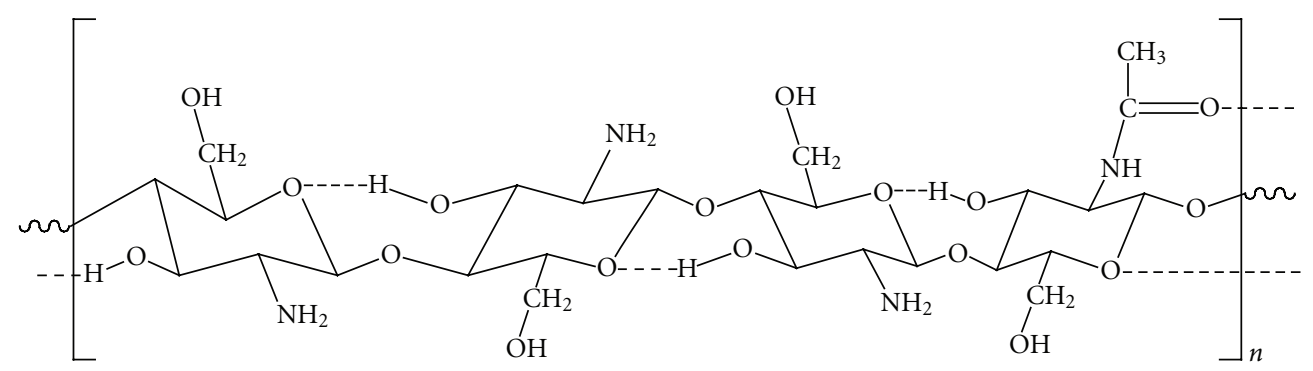

Figure 1: Structure of a chitosan with about $25 \%$ of acetylated repeating units.

regeneration was reported, because of their importance in the mobility and sensitivity of human body. The use of CHI, a completely bioresorbable material $[1,2]$, permits solving the main problems arising in the orthopaedic and neurological surgery: first, the substitution of damaged cartilage and bone with permanent prostheses of foreign biomaterials could not assure the same tribological and mechanical properties as the natural bone and cartilage, whose complete regeneration is preferable, when possible; second, the unique function of the nerve tissue can be fully restored only by regenerating it.

\section{Characterization of CHI-Based Biomaterials}

Regarding the analytical studies on CHI and its derivatives, some of them were carried out in view of a use of the polysaccharide in the biomedical field. Japanese researchers prepared and characterized polyion complexes formed by the reaction between $\mathrm{CHI}$ and the anionic polyelectrolyte gellan [15]; the complexes were structured in fibres and capsules, which were said to be able, when filled with either drugs or growth factors, to release them into the injured part of the body, during the biodegradation of the component biopolymers [16]. However, in a following study of the same authors, the biodegradation of the polyion complex fibres was found to occur via soil filamentous fungi, so that they were proposed as environmentally biodegradable materials [17]. This fact throws many doubts on the possible use of these fibres as bioresorbable materials within the body. The biochemistry, the histology, and the clinical uses of chitins and chitosans for the treatment of leg ulcers, the use of skin substitutes, and the regeneration of nerve, meniscus, and bone tissues were thoroughly reviewed by A. Muzzarelli et al. in 1999 [18]. Regarding the nerve regeneration, they cited an early paper of Zielinski and Aebischer, who found that the fibroblast cells R208N.8 released nerve growth factor, when sequestered in $60: 40$ acrylonitrile-vinyl chloride copolymer containing precipitated $\mathrm{CHI}$ as an internal matrix [19]. Regarding the meniscus, Muzzarelli et al. found that CHI stimulated its repair by providing the necessary tissue elements and humoral factors. Regarding the bone tissue, they reviewed all the work then made, both in vitro and in vivo. A short review by Babensee et al., regarding the growth factor delivery also from $\mathrm{CHI}$-albumin microspheres, for musculoskeletal, neural, and hepatic tissue engineering, appeared in the following year. However, CHI-albumin microspheres were used only for the growth of hepatocytes [20]; then, they were not useful for cartilage, bone, and nerve regeneration.

$\mathrm{CHI}$ was grafted onto silk fibroin by means of mushroom tyrosinase through the reaction of the amino groups of $\mathrm{CHI}$ with the tyrosyl residues of the protein, oxidized enzymatically to $o$-quinone groups; the resulting copolymer was analyzed, with the aim of enhancing the biocompatibility of silkbased biomedical devices in the hosting biological environment [21]. However, products of the same reaction, carried out under heterogeneous conditions, seemed to be, for their authors, more interesting as structural polymers than as biomedical ones [22]. The interactions with bovine serum albumin of two CHI macromolecules, having acetylation degrees of $1 \%$ and $12 \%$, were measured by means of the turbidity change with varying $\mathrm{pH}$, in $0.1 \mathrm{M} \mathrm{NaCl}$ solution. The results were presented as a model for enzyme immobilization on CHI, but no test with enzymes was reported [23]. Biodegradable blend membranes composed of $\mathrm{CHI}$, and either poly(D,L-lactide) or poly(L-lactide) was prepared using a solution-casting and solvent-extracting processing technique. The membranes were examined by means of SEM, FTIR, TGA, DSC, DMA, and X-ray diffraction to test the miscibility of the polymers, which depended strongly on the polymer concentration and on the composition ratio of the mixed solvents, as well as on the drying technique. In the blends prepared under optimized processing conditions, FTIR showed hydrogen bond interactions between the polymers, which also caused a lowering of their crystallinity, detected by X-ray, thermal and dynamical testing, so indicating a good miscibility [24]. Fluorescein was attached, via its epoxy derivative, to water-soluble $\mathrm{CHI}$, and the temperature/pH-sensitive qualities of fluorescence were investigated; the results obtained indicated that this modified $\mathrm{CHI}$ could be able to provide a convenient way to prepare low-cost and multifunctional macromolecular biomaterial for determining $\mathrm{pH}$ and temperature changes in biological systems simultaneously [25]. Bioartificial biodegradable materials were prepared, by the authors' group, by mixing CHI and poly(vinyl alcohol); then they were manufactured as films and finally cross-linked with pentane-2,5-dial (glutaraldehyde), both in the absence and in the presence of the edible plasticizer sorbitol, with the aim of using them as biomaterials and, in particular, as localized drug carriers. The materials were characterized by means of FTIR, DSC, TGA, X-ray diffraction, SEM, and tensile test 
[26]. The blends showed a good biodegradability [27] and, as toughened by dehydrothermal treatment, no cytotoxicity toward murine fibroblasts [28]; their ability for drug release and permeation, tested for ascorbic acid, paclitaxel, $\mathrm{D}(+)$ glucose, vitamin $\mathrm{B} 12$, and bovine serum albumin, was found to depend on the chemical structures and properties of the tested molecules $[27,28]$. The results obtained indicate that these blends could be useful to make both scaffolds for tissue engineering and devices for drug delivery. Canadian researchers fractionated and characterized $\mathrm{CHI}$ by size-exclusion chromatography and ${ }^{1} \mathrm{H}$ NMR, to produce homogeneous monodisperse chitosans in the molecular weight range of $5-100 \mathrm{kDa}$, which were said to be particularly useful in biomedical applications such as gene delivery; however, no results in the biomedical field were reported [29]. Japanese researchers modified an AFM probe by depositing on its tip, through a micropipette controlled by micromanipulator, first an acetone solution of poly(lactic-co-glycolic acid) and then a CHI solution in ethanol. The modified probe was used to study the interaction between the polymers and a mucin film, to understand the mucoadhesive mechanisms of $\mathrm{CHI}$, when used for oral mucosal drug delivery. It was revealed that when a poly (lactic-co-glycolic acid) probe is retracting from the mucin film, a repulsive force appeared; however, after the probe was further overcoated with $\mathrm{CHI}$, the force became attractive if the amount of $\mathrm{CHI}$ was enough, such as at $\mathrm{CHI}$ concentrations of $0.2 \% \mathrm{w} / \mathrm{v}$ [30]. Chinese researchers prepared a stable and translucent novel composite film consisting of $\mathrm{CHI}$ and cortical cells, extracted from waste wool fibres and characterized it by SEM, FTIR, DSC, X-ray diffraction, and tensile test. This film has a potential utilization in many fields, such as food packaging, wound dressing, and also tissue engineering [31]. Canadian researchers used AFM and AFM-based force spectroscopy, with CHI-modified tips, to investigate desorption of individual CHI polymer chains from substrates with varying chemical composition. They concluded that the experimental results reported in their paper might be used as a basis to investigate the interaction of CHI with surfaces, which may later be used as coatings in biomaterial applications, although no application in this field has been reported at the present time [32]. A new label-free amperometric immunosensor was developed for detection of human chorionic gonadotrophin, based on a multiwall carbon nanotubes-CHI complex film, electrodeposited on a glassy carbon electrode, and a three-dimensional Au nanoparticles- $\mathrm{TiO}_{2}$ hybrid. The ease of the nonmanual technique and the promising features of this composite were presented by these researchers as a possible versatile platform for constructing other biosensors [33]. Direct formation of porous $\mathrm{CHI}$ structures, to be used as scaffolds for cell culture, by supercritical carbon dioxide method was presented to an international conference in India in 2009 [34]. In the same year, a review appeared, regarding chitins and chitosans for the repair of wounded skin, nerve, cartilage, and bone [35]. Generally, these analytical studies pointed out the suitability of $\mathrm{CHI}$ and of its derivatives to be used in the biomedical field, mainly for tissue engineering and regeneration, as well as for drug and gene delivery.

\section{Bone and Cartilage Regeneration}

An early work in the field of the chondroskeletal tissue engineering regarded the use of a $\mathrm{CHI}$ ascorbate gel for the treatment of periodontitis, according to current dental surgery; in ten patients, two months after the treatment, $\mathrm{CHI}$ was completely reabsorbed and the periodontium well regenerated [36]. The positive results of this pioneering clinical application encouraged the researches on the use of $\mathrm{CHI}$ containing biomaterials for bone and cartilage regeneration.

3.1. Bone. Methyl pyrrolidinone $\mathrm{CHI}$ was used to favour the formation of new bone tissue within the large alveolar cavity, remaining after the avulsion of a wisdom tooth [37]. The use of imidazolyl and 2-methyl-imidazolyl CHI for bone lesion healing was tested on sheep femoral condyles [38]. A particularly interesting feature was the use of $\mathrm{CHI}$ and its derivatives for the treatment of osteoporosis, a possible physicochemical component which was recently studied by the authors' group [39]. The release, as a consequence of CHI biodegradation, of the bone morphogenetic protein BMP-7 (OP-1), linked to an $\mathrm{N}, \mathrm{N}$-dicarboxymethyl $\mathrm{CHI}$ matrix in the form of a polyelectrolyte complex, was tested on the femoral condyles of four female osteoporotic rats. After 30 days, a complete new bone formation was observed in the surgical bone defects [40]. In another research [41], the same osteoporotic rats were treated with only hydroxyapatite and two biological glasses, with rather scarce results, whilst a CHIhydroxyapatite composite was successfully inserted in femoral condyles of healthy New Zealand rabbits. N,N-dicarboxymethyl $\mathrm{CHI}$ was found to chelate calcium and phosphate ions, forming a gel, which favoured osteogenesis while promoting bone mineralization. Bone regeneration was observed in bone defects surgically made in sheep femoral condyle and trochanter [41, 42], as well as in human mandible after tooth avulsion and cyst removal [42]. Surgical lesions in rat condylus were treated with N,N-dicarboxymethyl CHI and the sodium salt of 6-oxychitin. Morphological data indicate that 6-oxychitin promoted the best osteoarchitectural reconstruction, even though healing was slower compared to that with N,N-dicarboxymethyl CHI. Complete healing was obtained with N,N-dicarboxymethyl CHI within three weeks [43]. A blend of CHI gel, ionically cross-linked with ascorbic acid, $\mathrm{ZnO}, \mathrm{CaO}$, crystalline carbonate-hydroxyapatite, and $\mathrm{NaF}$, was prepared and tested by chemical, physical, and crystallographic measurements, with the aim of obtaining an efficient dressing for use during regeneration of the periodontal barrier [44]. A review focused on the manufacture of CHI-inorganic composites, based on calcium carbonate, calcium phosphate, and silica, pointed out their importance in the field of blood compatible materials, bone substitutes, and cements for bone repair and regeneration [45]. Biodegradable polylactide/CHI blends were used to fabricate scaffolds with well-distributed and interconnected porous structures. The porosity and the pore 
dimension were monitored to obtain scaffolds suitable for applications in cartilage or bone tissue engineering [24, 46]. A more recent work regarded a good influence of the CHI component on the interactions between those blends and rat osteoblasts [47]; however, at the present time no clinical application of them has been reported. The bioactivity of a novel composite of carbonate-containing low-crystallinity nanoparticle hydroxyapatite and a CHI-phosphorylated CHI polyelectrolyte complex was evaluated in vitro and in vivo. The material was cocultured with rat osteoblasts in vitro and implanted into rabbit femur marrow cavities. The results indicated that the composite promoted osteoblast adhesion, morphology, proliferation, and differentiation in vitro; the bone tissue response in vivo to the material showed that the composite provided a suitable environment for active bone formation, with marrow cell infiltration and new bone deposition around the powder; then, it was bioactive as well as biodegradable [48]. Chitin and CHI were used to fill the defects in fractured segments of radius and ulna of dogs after stabilizing with dynamic compression plates. The study revealed that the fracture healing was better in $\mathrm{CHI}$ group of dogs [49]. Taiwan researchers produced a CHI surface-bonded recombinant human bone morphogenetic protein 2 via amide bond formation between the components. The surface-bonded protein did not denature but expressed sustained biological activity, such as osteoblast cell adhesion, proliferation, and differentiation, so making the material useful for bone tissue regeneration [50]. Korean researchers prepared porous, biodegradable and biocompatible scaffolds, using CHI, CHI with natural hydroxyapatite derived from Thunnus Obesus bone, and CHI grafted with functionalized multiwalled carbon nanotube, via freeze-drying method, and characterized them physiochemically as bone graft substitutes. Cell proliferation in composite scaffolds was twice than in pure CHI when checked in vitro using a human osteosarcoma cell line [51]. The preparation and characterization of CHI-blended polyamide- 6 nanofibres by a new single solvent system via electrospinning process for human osteoblastic cell culture applications were carried out. The in vitro cytotoxicity evaluation of these nanofibres indicated that this scaffold material was nontoxic for the osteoblast cell culture [52]. A Brazilian research group synthesised a porous chitosan-gelatin scaffold cross-linked by glutaraldehyde and characterized it by both physicochemical and morphological tests, as well as investigating its effects on growth and osteogenic differentiation of rat bone marrow mesenchymal stem cells. Free-cell scaffolds were implanted into tooth sockets of Lewis rats after upper first molars extraction; on the 21st day, alveolar bone and epithelial healing were completely established [53]. Recently, Muzzarelli reviewed the use, for bone regeneration, of CHI composites with inorganic materials, morphogenetic proteins, and stem cells [54]. As a whole, despite the numerous positive results obtained from the tests made both in vitro and in animals, at the present time CHI-containing biomaterials have not yet reached the clinical application for human bone tissue regeneration, with the only exception of dental surgery. Also a very recent Russian patent, regarding a porous sponge of chitosan- gelatin composite with octacalcium phosphate, suitable for filling of bone defects, does not contain, in its abstract, any mention of having obtained the government approval for its use in clinical orthopaedic practice [55].

3.2. Cartilage. N-Carboxybutyl CHI was used to promote the tissue repair of the meniscus in rabbits [56]. Recombinant bone morphogenetic protein BMP-7 (OP-1), linked to a N,N-dicarboxymethyl CHI matrix in the form of a polyelectrolyte complex, was used to induce or facilitate the repair of articular cartilage lesions, produced in 21 adult male New Zealand white rabbits [57]. In situ gelling CHI-disodium $\beta$-glycerol phosphate-glucosamine solution, containing chondrocytes, was injected into cartilage defects in rabbits. The results showed that the delivered cells could gradually produce a viable and mechanically stable repair tissue at the defect site [58]. Another group inserted a hydrogel of CHI-hyaluronan polyelectrolyte complex into the patella articular cartilage of rabbits, obtaining some promising results. The implants were capable of developing hyalineappearing tissue, maintained at 24 weeks postoperatively; the presence of chondrocytes was also observed [59]. CHI-glycerol phosphate/blood implants applied in conjunction with drilling, compared to drilling alone, elicited a more hyaline and integrated repair tissue associated with a porous subchondral bone repleted with blood vessels, in New Zealand white rabbits. Concomitant regeneration of a vascularized bone plate during cartilage repair could provide progenitors, anabolic factors, and nutrients that aid in the formation of hyaline cartilage [60]. An analogous treatment, made on identical rabbits subjected to bilateral arthrotomies, with each trochlea receiving a cartilage defect bearing four microdrill holes into the subchondral bone, favored intramembranous bone formation in the microdrill holes and resulted in a cartilage repair strategy that modulates acute and intermediate events in the subchondral bone in order to improve final cartilage repair outcome [61]. The proliferation in vitro of New Zealand white rabbit chondrocytes on porous poly(DL-lactide)/chitosan scaffolds was studied using scanning electron microscopy, histological observations, and proteoglycan measurements; the results indicated that the resulting scaffolds exhibited increasing ability to promote the attachment and proliferation of chondrocytes and also helped the seeded chondrocytes to spread through the scaffolds and distribute homogeneously inside them [62]. Korean researchers obtained thermosensitive gels grafting $\mathrm{N}$-isopropylacrylamide onto $\mathrm{CHI}$ and coupling $\mathrm{CHI}$ with Pluronic, a commercial poly(ethylene oxide- $b$-propylene oxide) triblock copolymer [63]. The first copolymer induced the chondrogenic differentiation in vitro of human mesenchymal stem cells [63]; the second one favoured the proliferation in vitro of bovine chondrocytes [64]. Temperatureresponsive $\mathrm{CHI}$ hydrogels were prepared by combining $\mathrm{CHI}, \beta$-sodium glycerophosphate, and hydroxyethyl cellulose. Tissue-engineered cartilage-regenerating scaffolds were made in vitro by mixing sheep chondrocytes with a $\mathrm{CHI}$ hydrogel. To collect data for in vivo repair, scaffolds cultured for one day were transplanted to the freshly prepared defects 
of the articular cartilage of sheep. The results showed that the chondrocytes in the regenerated cartilage survived and retained their ability to secrete matrix when cultured in vitro. The scaffolds induced complete cartilage defects repair within 24 weeks after being transplanted in vivo [65]. Canadian researchers successfully investigated temporal and spatial modulation of chondrogenic foci in subchondral microdrill holes, made in 32 New Zealand white rabbits, by CHI-glycerol phosphate/blood implants. The chondrogenic foci bore some similarities to growth cartilage and could give rise to a repair tissue having similar zonal stratification as articular cartilage [66]. In the field of cartilage regeneration, the research on CHI is less advanced than in that of bone, considering the absence of clinical applications in humans.

\section{Nerve Tissue Regeneration}

Chinese researchers studied the ability of materials made with $\mathrm{CHI}$ and $\mathrm{CHI}$ derivatives to facilitate the in vitro growth of nerve cells for nerve tissue regeneration. Their results suggested that, after being precoated with laminin and fibronectin solution or serum, all materials have better nerve cell affinity [67]. More recently, the same group found that films of carboxymethyl chitosan, cross-linked with 1-ethyl3(3-dimethylaminopropyl) carbodiimide hydrochloride, enhanced the spread of Neuro-2a cells and provided a good proliferation substratum for Neuro-2a cells, as compared to chitosan films [68]. In a much later research, that group investigated the application of the $\mathrm{CHI} /$ glycerol- $\beta$-phosphate disodium salt hydrogel in peripheral nerve regeneration, in 24 female adult Sprague Dawley rats. Contrary to former in vitro reports, they found that the implanted hydrogel actually impeded nerve regeneration; then, many further studies are necessary in this matter [69]. Japanese researchers coated apatite CHI tubes prepared from crab tendons and then used them as nerve-regenerating guides for the sciatic nerves of male Sprague Dawley rats, with successful results [70]. Researcher of the University of Texas reviewed the strategies for repair and regeneration of damaged nerves, among which there was the use of nerve guides made by bioresorbable materials, including CHI [71]. Chinese researchers developed a dual-component artificial nerve graft comprising an outer microporous conduit of $\mathrm{CHI}$, made from crab tendons, and internal oriented filaments of poly(glycolic acid). The novel graft was used for bridging sciatic nerve across a defect of $3 \mathrm{~cm}$ length in six Beagle dogs; they were compared with other six dogs subjected to autograft, as a positive control, and with five not grafted dogs, as a negative control. At six months postoperatively, the dogs grafted with the artificial nerve showed motion ability comparable to that of the positive control ones, unlike the not grafted ones [72]. Italian researchers made in vitro neuroblast adhesion test on films of CHI-gelatin blends and on nerve guides of CHI-poly( $\varepsilon$-caprolactone) blends; the cells adhered better to the first materials than to the second ones [73]. A US research team prepared nerve guides with a blend of CHI and type I collagen from rat-tail tendon, as well as guides of pure CHI. Each guide group was used to bridge a $10 \mathrm{~mm}$ sciatic nerve gap of 24 female Lewis rats; equal numbers of rats were subjected to autograft and left unrepaired, respectively, as positive and negative controls. Both guides gave quite good results, although less than autograft; the researchers concluded that further investigation was necessary [74]. A Chinese group fabricated nerve conduits of a CHI-poly(lactic acid) blend, using a mold casting/infrared dehydration technique. The conduits were inserted in $10 \mathrm{~mm}$ gaps of the sciatic nerves of ten 4-month-old Sprague Dawley rats; equal numbers of rats were treated with autograft and silicone conduits, respectively. The nerve regeneration with the blend was not different from that with the autograft, and much better than with the silicone [75]. A group of the Purdue University, USA, performed ex vivo and in vivo experiments on spinal cord injuries made in guinea pigs. Their results demonstrated that the application of $\mathrm{CHI}$ was able to immediately restore compromised membrane integrity, $\mathrm{CHI}$ was a potent neuroprotective agent, even though it did not show any ability to scavenge either reactive oxygen species or acrolein, and that CHI clearly targeted the area of tissue damage, where uninjured spinal cord exhibited a very weak affinity for $\mathrm{CHI}$. Then, the CHI approach for damaged membranes provides novel therapeutic potential through site-specific delivery following traumatic spinal cord and head injury [76]. Canadian researchers found that $\mathrm{CHI}$ could be promising in transplantation strategies of neural stem and progenitor cells, to treat an injury to the central nervous system, such as a spinal cord injury. Four amine-functionalized hydrogels, comprised $\mathrm{CHI}$, were screened in vitro for the viability, the migration, and the differentiation of adult murine neural stem and progenitor cells. Only CHI supported survival of multipotent stem cells and the differentiation of the progenitor cells into neurons, astrocytes, and oligodendrocytes. Then, CHI appeared as a promising material for the therapies involving adult neural stem and progenitor cells [77]. Conductive polycaprolactone/CHI/polypyrrole composites were prepared and characterized in view of their possible use for peripheral nerve repair [78]; conductive conduits made with polypyrrole particles dispersed in a $\mathrm{CHI}-g$-polycaprolactone matrix were prepared and characterized by the same group, then implanted into rabbits for various periods, to test the variations of their properties during bioresorption in vivo [79]; however, no data about a test of them as nerve repair conduits have been reported. To design a novel kind of scaffolds for blood vessel and nerve repairs, random and aligned nanofibrous scaffolds based on collagen-CHI-thermoplastic polyurethane blends were electrospun to mimic the componential and structural aspects of the native extracellular matrix. Vascular grafts and nerve conduits were electrospun or sutured based on the nanofibrous scaffolds; the results indicated that nanofibrous scaffolds, made blending collagen, $\mathrm{CHI}$, and thermoplastic polyurethane might be a potential candidate for vascular repair and nerve regeneration [80]. Another group developed an aligned CHI-polycaprolactone fibrous scaffold and investigated how the fibre alignment influenced nerve cell organization and function in comparison with randomly oriented fibrous scaffolds and cast films of the same material. Schwann cells grown on the aligned CHI-polycaprolactone fibres exhibited a 
TABLE 1: CHI-containing devices used for bone and nerve tissue regeneration in humans.

\begin{tabular}{lccc}
\hline Device & Tissue & Time & Reference \\
\hline CHI ascorbate gel & periodontium & 2 months & {$[35]$} \\
Me-pyrrolidinone-CHI gel & tooth alveolar bone & 6 months & 15 days \\
DCMC-CaPa gel or solution & mandible bone & $36]$ & {$[41]$} \\
CHI conduits with PGA ${ }^{b}$ filaments & right arm median nerve & 36 months & {$[86]$} \\
\hline
\end{tabular}

${ }^{a} \mathrm{~N}, \mathrm{~N}$-dicarboxymethyl CHI with calcium phosphate;

${ }^{\mathrm{b}}$ poly(glycolic acid).

bipolar morphology that oriented along the fibre alignment direction, while those on the films and randomly oriented fibres had a multipolar morphology. Similarly, the CHI-polycaprolactone material supported neuron-like PC-12 cell adhesion, and the aligned fibres regulated the growth of PC-12 cells along the fibre orientation. Additionally, PC-12 cells cultured on the aligned fibres exhibited enhanced unidirectional neurite extension along fibre orientation and significantly higher $\beta$-tubulin gene expression than those grown on CHI-polycaprolactone films and randomly oriented fibres. The results reported suggest that the aligned $\mathrm{CHI}$-polycaprolactone fibres can serve as a suitable scaffold for improved nerve tissue reconstruction [81]. The differentiation of bone marrow stromal cells in three-dimensional scaffolds consisting of collagen, poly(lactide-co-glycolide), and $\mathrm{CHI}$ was also investigated. The induction with neuron growth factor inhibited osteogenesis and guided the differentiation of bone marrow stromal cells towards neurons in the constructs. Therefore, the combination of collagen-functionalized poly(lactide-co-glycolide)/CHI scaffolds, neuron growth factor, and bone marrow stromal cells can be promising in neural tissue engineering [82]. Indeed, bone marrow stromal cells are generally known to be useful for bone tissue regeneration [83]. To have found a possible use of them also for nerve tissue regeneration opens new ways to neural surgery.

Regarding the aim of using bioresorbable macromolecular materials to make nerve regeneration conduits to be employed in the clinical practice of human neural surgery, it has been reached since quite several years, both with only synthetic bioresorbable polyesters $[84,85]$ and with blends of them with $\mathrm{CHI}$ [86]. In the latter case, a 37-year-old Chinese man, having a $35 \mathrm{~mm}$-long nerve defect in the median nerve of the right arm at elbow level, underwent a surgical repair with an artificial nerve graft, comprising an outer microporous conduit of $\mathrm{CHI}$ and internal oriented filaments of poly(glycolic acid). Suitable functional recovery of the hand ability proceeded slowly, but regularly, with time, together with nerve regeneration, and was near complete 36 months after the implantation [86]. The same Chinese surgeon group reviewed the construction of tissue-engineered nerve grafts and their application in peripheral nerve regeneration very recently [87]. As regarding the use of $\mathrm{CHI}$-containing grafts in clinical neural surgery, only their preceding intervention was reported, in comparison with many equally successful ones with other biomaterials. This fact might be a sign that CHI-containing nerve grafts still need much theoretical and laboratory study before becoming of common practice in surgery.

\section{Conclusion}

The importance of the biomaterials containing either $\mathrm{CHI}$ or $\mathrm{CHI}$ derivatives for the regeneration of damaged bone, cartilage, and nerve tissue appears evident from the studies carried out near towards the entire world. However, as it can be seen from the data summarized in Table 1, only few applications to the human health in this field have been done at the present time. Then, much work must still be made, especially for the passage from the experimentation of the CHI-based devices in vitro and in animals, in which many successful results were obtained, to their general application in clinical practice.

\section{References}

[1] J. Berger, M. Reist, J. M. Mayer, O. Felt, N. A. Peppas, and R. Gurny, "Structure and interactions in covalently and ionically crosslinked chitosan hydrogels for biomedical applications," European Journal of Pharmaceutics and Biopharmaceutics, vol. 57, no. 1, pp. 19-34, 2004.

[2] C. Tangsadthakun, S. Kanokpanont, N. Sanchavanakit et al., "The influence of molecular weight of chitosan on the physical and biological properties of collagen/chitosan scaffolds," Journal of Biomaterials Science, vol. 18, no. 2, pp. 147163, 2007.

[3] R. A. A. Muzzarelli, "Immobilization of enzymes on chitin and chitosan," Enzyme and Microbial Technology, vol. 2, no. 3, pp. 177-184, 1980.

[4] P. Cerrai, G. D. Guerra, M. Tricoli, S. Maltinti, N. Barbani, and L. Petarca, "Polyelectrolyte complexes obtained by radical polymerization in the presence of chitosan," Macromolecular Chemistry and Physics, vol. 197, no. 11, pp. 3567-3579, 1996.

[5] G. D. Guerra, P. Cerrai, M. Tricoli, S. Maltinti, and R. S. Del Guerra, "In vitro cytotoxicity testing of chitosancontaining polyelectrolyte complexes," Journal of Materials Science: Materials in Medicine, vol. 9, no. 2, pp. 73-76, 1998.

[6] R. A. A. Muzzarelli, G. Barontini, and R. Rocchetti, "Immobilized enzymes on chitosan columns: $\alpha$ chymotrypsin and acid phosphatase," Biotechnology and Bioengineering, vol. 18, no. 10, pp. 1445-1454, 1976.

[7] R. A. A. Muzzarelli, G. Barontini, and R. Rocchetti, "Isolation of lysozyme on chitosan," Biotechnology and Bioengineering, vol. 20, no. 1, pp. 87-94, 1978.

[8] R. A. A. Muzzarelli and O. Tubertini, "Chitin and chitosan as chromatographic supports and adsorbents for collection of 
metal ions from organic and aqueous solutions and sea-water," Talanta, vol. 16, no. 12, pp. 1571-1577, 1969.

[9] R. A. A. Muzzarelli and R. Rocchetti, "The use of chitosan columns for the removal of mercury from waters," Journal of Chromatography A, vol. 96, no. 1, pp. 115-121, 1974.

[10] R. A. Muzzarelli, A. Isolati, and A. Ferrero, "Chitosan membranes," Ion Exchange and Membranes, vol. 1, no. 4, pp. 193196, 1974.

[11] R. A. A. Muzzarelli, C. Zucchini, P. Ilari et al., "Osteoconductive properties of methyl-pyrrolidinone chitosan in an animal model," Biomaterials, vol. 14, no. 12, pp. 925-929, 1993.

[12] K. H. Bodek and A. Kufelnicki, "Interaction of microcrystalline chitosan with $\mathrm{Ni}(\mathrm{II})$ and $\mathrm{Mn}$ (II) ions in aqueous solution," Journal of Applied Polymer Science, vol. 98, no. 6, pp. 2572-2577, 2005

[13] R. A. A. Muzzarelli and C. Muzzarelli, "Chitosan chemistry: relevance to the biomedical sciences," Advances in Polymer Science, vol. 186, pp. 151-209, 2005.

[14] I. Y. Kim, S. J. Seo, H. S. Moon et al., "Chitosan and its derivatives for tissue engineering applications," Biotechnology Advances, vol. 26, no. 1, pp. 1-21, 2008.

[15] M. Amaike, Y. Senoo, and H. Yamamoto, "Sphere, honeycomb, regularly spaced droplet and fiber structures of polyion complexes of chitosan and gellan," Macromolecular Rapid Communications, vol. 19, no. 6, pp. 287-289, 1998.

[16] H. Yamamoto and Y. Senoo, "Polyion complex fiber and capsule formed by self-assembly of chitosan and gellan at solution interfaces," Macromolecular Chemistry and Physics, vol. 201, no. 1, pp. 84-92, 2000.

[17] K. Ohkawa, M. Yamada, A. Nishida, N. Nishi, and H. Yamamoto, "Biodegradation of chitosan-gellan and poly(Llysine)-gellan polyion complex fibers by pure cultures of soil filamentous fungi," Journal of Polymers and the Environment, vol. 8, no. 2, pp. 59-66, 2000.

[18] R. A. A. Muzzarelli, M. Mattioli-Belmonte, A. Pugnaloni, and G. Biagini, "Biochemistry, histology and clinical uses of chitins and chitosans in wound healing," in Chitin and Chitinases, P. Jollès and R. A. A. Muzzarelli, Eds., pp. 251-264, Birkhäuser, Basel, Switzerland, 1999.

[19] B. A. Zielinski and P. Aebischer, "Chitosan as a matrix for mammalian cell encapsulation,” Biomaterials, vol. 15, no. 13, pp. 1049-1056, 1994.

[20] J. E. Babensee, L. V. McIntire, and A. G. Mikos, "Growth factor delivery for tissue engineering," Pharmaceutical Research, vol. 17, no. 5, pp. 497-504, 2000.

[21] S. Sampaio, P. Taddei, P. Monti, J. Buchert, and G. Freddi, "Enzymatic grafting of chitosan onto Bombyx mori silk fibroin: kinetic and IR vibrational studies," Journal of Biotechnology, vol. 116, no. 1, pp. 21-33, 2005.

[22] G. Freddi, A. Anghileri, S. Sampaio, J. Buchert, P. Monti, and P. Taddei, "Tyrosinase-catalyzed modification of Bombyx mori silk fibroin: grafting of chitosan under heterogeneous reaction conditions," Journal of Biotechnology, vol. 125, no. 2, pp. 281294, 2006.

[23] A. B. Kayitmazer, D. Shaw, and P. L. Dubin, "Role of polyelectrolyte persistence length in the binding of oppositely charged micelles, dendrimers, and protein to chitosan and poly(dimethyldiallyammonium chloride)," Macromolecules, vol. 38, no. 12, pp. 5198-5204, 2005.

[24] Y. Wan, H. Wu, A. Yu, and D. Wen, "Biodegradable polylactide/chitosan blend membranes," Biomacromolecules, vol. 7, no. 4 , pp. 1362-1372, 2006.
[25] X. Guan, X. Liu, and Z. Su, "Preparation and photophysical behaviors of fluorescent chitosan bearing fluorescein: potential biomaterial as temperature/pH probes," Journal of Applied Polymer Science, vol. 104, no. 6, pp. 3960-3966, 2007.

[26] C. Cristallini, G. D. Guerra, N. Barbani, and F. Bianchi, "Biodegradable bioartificial materials made with chitosan and poly(vinyl alcohol). Part I: physicochemical characterization," Journal of Applied Biomaterials and Biomechanics, vol. 5, no. 3, pp. 184-191, 2007.

[27] C. Cristallini, N. Barbani, F. Bianchi, D. Silvestri, and G. D. Guerra, "Biodegradable bioartificial materials made by chitosan and poly(vinyl alcohol). Part II: enzymatic degradability and drug-releasing ability," Biomedical Engineering Applications, Basis and Communications, vol. 20, no. 5, pp. 321-328, 2008.

[28] D. Silvestri, N. Barbani, G. D. Guerra, M. Gagliardi, and C. Cristallini, "Biodegradable bioartificial materials made by chitosan and poly(vinyl alcohol) part III: materials toughened by means of a dehydrothermal treatment," Biomedical Engineering Applications, Basis and Communications, vol. 22, no. 6, pp. 509-517, 2010.

[29] S. Nguyen, S. Hisiger, M. Jolicoeur, F. M. Winnik, and M. D. Buschmann, "Fractionation and characterization of chitosan by analytical SEC and 1H NMR after semi-preparative SEC," Carbohydrate Polymers, vol. 75, no. 4, pp. 636-645, 2009.

[30] D. Li, H. Yamamoto, H. Takeuchi, and Y. Kawashima, "A novel method for modifying AFM probe to investigate the interaction between biomaterial polymers (Chitosan-coated PLGA) and mucin film," European Journal of Pharmaceutics and Biopharmaceutics, vol. 75, no. 2, pp. 277-283, 2010.

[31] J. Fan and W. D. Yu, "Biomaterials from wool wastes: characterization of cortical cells/chitosan composite," Waste Management and Research, vol. 28, no. 1, pp. 44-50, 2010.

[32] M. Kocun, M. Grandbois, and L. A. Cuccia, "Single molecule atomic force microscopy and force spectroscopy of chitosan," Colloids and Surfaces B: Biointerfaces, vol. 82, no. 2, pp. 470476, 2011.

[33] H. Yang, R. Yuan, Y. Chai, and Y. Zhuo, "Electrochemically deposited nanocomposite of chitosan and carbon nanotubes for detection of human chorionic gonadotrophin," Colloids and Surfaces B: Biointerfaces, vol. 82, no. 2, pp. 463-469, 2011.

[34] S. Tripathi, K. Rinki, P. K. Dutta, A. J. Hunt, D. J. Macquarrie, and J. H. Clark, "Direct chitosan scaffold formation via chitin whiskers by supercritical carbon dioxide method: a green approach," in Proceedings of the 1st International Conference on Nanostructured Materials and Nanocomposites (ICNM '09), pp. 234-241, Applied Science Innovations Private Limited, Kottayam, India, 2009.

[35] R. A. A. Muzzarelli, "Chitins and chitosans for the repair of wounded skin, nerve, cartilage and bone," Carbohydrate Polymers, vol. 76, no. 2, pp. 167-182, 2009.

[36] R. Muzzarelli, G. Biagini, A. Pugnaloni et al., "Reconstruction of parodontal tissue with chitosan," Biomaterials, vol. 10, no. 9, pp. 598-603, 1989.

[37] R. A. A. Muzzarelli, G. Biagini, M. Bellardini, L. Simonelli, C. Castaldini, and G. Fratto, "Osteoconduction exerted by methylpyrrolidinone chitosan used in dental surgery," Biomaterials, vol. 14, no. 1, pp. 39-43, 1993.

[38] R. A. A. Muzzarelli, M. Mattioli-Belmonte, C. Tietz et al., "Stimulatory effect on bone formation exerted by a modified chitosan," Biomaterials, vol. 15, no. 13, pp. 1075-1081, 1994.

[39] N. Barbani, E. Rosellini, C. Cristallini, G. D. Guerra, A. Krajewski, and M. Mazzocchi, "Hydroxyapatite-collagen composites. Part I: can the decrease of the interactions between 
the two components be a physicochemical component of osteoporosis in aged bone?" Journal of Materials Science: Materials in Medicine, vol. 22, no. 3, pp. 637-646, 2011.

[40] R. A. A. Muzzarelli, G. Biagini, M. Mattioli Belmonte et al., "Osteoinduction by chitosan-complexed BMP: morphostructural responses in an osteoporotic model," Journal of Bioactive and Compatible Polymers, vol. 12, no. 4, pp. 321-329, 1997.

[41] M. Mattioli Belmonte, A. De Benedittis, R. A. A. Muzzarelli et al., "Bioactivity modulation of bioactive materials in view of their application in osteoporotic patients," Journal of Materials Science: Materials in Medicine, vol. 9, no. 9, pp. 485-492, 1998.

[42] R. A. A. Muzzarelli, V. Ramos, V. Stanic et al., "Osteogenesis promoted by calcium phosphate N,N-dicarboxymethyl chitosan," Carbohydrate Polymers, vol. 36, no. 4, pp. 267-276, 1998.

[43] M. Mattioli-Belmonte, N. Nicoli-Aldini, A. De Benedittis et al., "Morphological study of bone regeneration in the presence of 6-oxychitin," Carbohydrate Polymers, vol. 40, no. 1, pp. 2327, 1999.

[44] M. Mattioli Belmonte, A. De Benedittis, R. Mongiorgi et al., "Bioactivity of chitosan in dentistry. Preliminary data on chitosan-based cements," Minerva Stomatologica, vol. 48, no. 12, pp. 567-576, 1999

[45] C. Muzzarelli and R. A. A. Muzzarelli, "Natural and artificial chitosan-inorganic composites," Journal of Inorganic Biochemistry, vol. 92, no. 2, pp. 89-94, 2002.

[46] Y. Wan, Y. Fang, H. Wu, and X. Cao, "Porous polylactide/ chitosan scaffolds for tissue engineering," Journal of Biomedical Materials Research A, vol. 80, no. 4, pp. 776-789, 2007.

[47] H. Wu, Y. Wan, S. Dalai, and R. Zhang, "Response of rat osteoblasts to polycaprolactone/chitosan blend porous scaffolds," Journal of Biomedical Materials Research A, vol. 92, no. 1, pp. 238-245, 2010.

[48] Q. L. Li, M. Y. Wu, L. L. Tang, J. Zhou, Y. Jiang, and B. W. Darvell, "Bioactivity of a novel nanocomposite of hydroxyapatite and chitosan-phosphorylated chitosan polyelectrolyte complex," Journal of Bioactive and Compatible Polymers, vol. 23, no. 6, pp. 520-531, 2008.

[49] G. D. J. Rao, N. N. Balasubramanian, S. Prathaban, and B. J. William, "Clinical evaluation of chitin and chitosan in the management of fractures," Tamilnadu Journal of Veterinary and Animal Sciences, vol. 5, no. 1, pp. 1-3, 2009.

[50] C. H. Chang, C. T. Tsao, K. Y. Chang et al., "Chitosan membrane with surface-bonded growth factor in guided tissue regeneration applications," Journal of Bioactive and Compatible Polymers, vol. 25, no. 5, pp. 465-482, 2010.

[51] J. Venkatesan, Z. J. Qian, B. Ryu, N. A. Kumar, and S. K. Kim, "Preparation and characterization of carbon nanotubegrafted-chitosan-natural hydroxyapatite composite for bone tissue engineering," Carbohydrate Polymers, vol. 83, no. 2, pp. 569-577, 2011.

[52] R. Nirmala, R. Navamathavan, H. S. Kang, M. H. El-Newehy, and H. Y. Kim, "Preparation of polyamide-6/chitosan composite nanofibers by a single solvent system via electrospinning for biomedical applications," Colloids and Surfaces B, vol. 83, no. 1, pp. 173-178, 2011.

[53] S. C. C. C. Miranda, G. A. B. Silva, R. C. R. Hell, M. D. Martins, J. B. Alves, and A. M. Goes, "Three-dimensional culture of rat BMMSCs in a porous chitosan-gelatin scaffold: a promising association for bone tissue engineering in oral reconstruction," Archives of Oral Biology, vol. 56, no. 1, pp. 1-15, 2011.
[54] R. A. A. Muzzarelli, "Chitosan composites with inorganics, morphogenetic proteins and stem cells, for bone regeneration," Carbohydrate Polymers, vol. 83, no. 4, pp. 1433-1445, 2011.

[55] S. M. Barinov, V. S. Komlev, I. V. Fadeeva, A. Y. Fedotov, and A. S. Fomin, "Porous composite chitosan and gelatin-based material for filling of bone defects," Russ., CODEN: RUXXE7 RU 2412711 C1 20110227 Application: RU 2009-134778 20090917. Priority: RU 2009-134778 20090917. Patent written in Russian. CAN 154:268927, 6 pages, 2011.

[56] R. Muzzarelli, V. Bicchiega, G. Biagini, A. Pugnaloni, and R. Rizzoli, "Role of N-Carboxybutyl chitosan in the repair of the meniscus," Journal of Bioactive and Compatible Polymers, vol. 7, no. 2, pp. 130-148, 1992.

[57] M. Mattioli-Belmonte, A. Gigante, R. A. A. Muzzarelli et al., "N,N-dicarboxymethyl chitosan as delivery agent for bone morphogenetic protein in the repair of articular cartilage," Medical and Biological Engineering and Computing, vol. 37, no. 1, pp. 130-134, 1999.

[58] C. D. Hoemann, J. Sun, A. Légaré, M. D. McKee, and M. D. Buschmann, "Tissue engineering of cartilage using an injectable and adhesive chitosan-based cell-delivery vehicle," Osteoarthritis and Cartilage, vol. 13, no. 4, pp. 318-329, 2005.

[59] S. R. Frenkel, G. Bradica, J. H. Brekke et al., "Regeneration of articular cartilage-evaluation of osteochondral defect repair in the rabbit using multiphasic implants," Osteoarthritis and Cartilage, vol. 13, no. 9, pp. 798-807, 2005.

[60] C. D. Hoemann, J. Sun, M. D. McKee et al., "Chitosan-glycerol phosphate/blood implants elicit hyaline cartilage repair integrated with porous subchondral bone in microdrilled rabbit defects," Osteoarthritis and Cartilage, vol. 15, no. 1, pp. 78-89, 2007.

[61] A. Chevrier, C. D. Hoemann, J. Sun, and M. D. Buschmann, "Chitosan-glycerol phosphate/blood implants increase cell recruitment, transient vascularization and subchondral bone remodeling in drilled cartilage defects," Osteoarthritis and Cartilage, vol. 15, no. 3, pp. 316-327, 2007.

[62] H. Wu, Y. Wan, X. Cao, and Q. Wu, "Proliferation of chondrocytes on porous poly(dl-lactide)/chitosan scaffolds," Acta Biomaterialia, vol. 4, no. 1, pp. 76-87, 2008.

[63] H. J. Chung, J. W. Bae, H. D. Park, J. W. Lee, and K. D. Park, "Thermosensitive chitosans as novel injectable biomaterials," Macromolecular Symposia, vol. 224, no. 1, pp. 275-286, 2005.

[64] K. M. Park, S. Y. Lee, Y. K. Joung, J. S. Na, M. C. Lee, and K. D. Park, "Thermosensitive chitosan-Pluronic hydrogel as an injectable cell delivery carrier for cartilage regeneration," Acta Biomaterialia, vol. 5, no. 6, pp. 1956-1965, 2009.

[65] T. Hao, N. Wen, J. K. Cao et al., "The support of matrix accumulation and the promotion of sheep articular cartilage defects repair in vivo by chitosan hydrogels," Osteoarthritis and Cartilage, vol. 18, no. 2, pp. 257-265, 2010.

[66] A. Chevrier, C. D. Hoemann, J. Sun, and M. D. Buschmann, "Temporal and spatial modulation of chondrogenic foci in subchondral microdrill holes by chitosan-glycerol phosphate/blood implants," Osteoarthritis and Cartilage, vol. 19, no. 1, pp. 136-144, 2011.

[67] H. Gong, Y. Zhong, J. Li, Y. Gong, N. Zhao, and X. Zhang, "Studies on nerve cell affinity of chitosan-derived materials," Journal of Biomedical Materials Research, vol. 52, no. 2, pp. 285-295, 2000.

[68] G. Lu, L. Kong, B. Sheng, G. Wang, Y. Gong, and X. Zhang, "Degradation of covalently cross-linked carboxymethyl chitosan and its potential application for peripheral nerve 
regeneration," European Polymer Journal, vol. 43, no. 9, pp. 3807-3818, 2007.

[69] L. Zheng, Q. Ao, H. Han, X. Zhang, and Y. Gong, "Evaluation of the chitosan/glycerol- $\beta$-phosphate disodium salt hydrogel application in peripheral nerve regeneration," Biomedical Materials, vol. 5, no. 3, Article ID 035003, 2010.

[70] I. Yamaguchi, S. Itoh, M. Suzuki, A. Osaka, and J. Tanaka, "The chitosan prepared from crab tendons: II. The chitosan/apatite composites and their application to nerve regeneration," Biomaterials, vol. 24, no. 19, pp. 3285-3292, 2003.

[71] C. E. Schmidt and J. B. Leach, "Neural tissue engineering: strategies for repair and regeneration," Annual Review of Biomedical Engineering, vol. 5, pp. 293-347, 2003.

[72] X. Wang, W. Hu, Y. Cao, J. Yao, J. Wu, and X. Gu, "Dog sciatic nerve regeneration across a $30-\mathrm{mm}$ defect bridged by a chitosan/PGA artificial nerve graft," Brain, vol. 128, no. 8, pp. 1897-1910, 2005.

[73] G. Ciardelli and V. Chiono, "Materials for peripheral nerve regeneration," Macromolecular Bioscience, vol. 6, no. 1, pp. 1326, 2006.

[74] M. Patel, P. J. VandeVord, H. W. Matthew, S. De Silva, B. Wu, and P. H. Wooley, "Collagen-chitosan nerve guides for peripheral nerve repair: a histomorphometric study," Journal of Biomaterials Applications, vol. 23, no. 2, pp. 101-121, 2008.

[75] F. Xie, Q. F. Li, B. Gu, K. Liu, and G. X. Shen, "In vitro and in vivo evaluation of a biodegradable chitosan-PLA composite peripheral nerve guide conduit material," Microsurgery, vol. 28, no. 6, pp. 471-479, 2008.

[76] Y. Cho, R. Shi, and R. B. Borgens, "Chitosan produces potent neuroprotection and physiological recovery following traumatic spinal cord injury," Journal of Experimental Biology, vol. 213, no. 9, pp. 1513-1520, 2010.

[77] V. I. Scanga, A. Goraltchouk, N. Nussaiba, M. S. Shoichet, and C. M. Morshead, "Biomaterials for neural-tissue engineering - chitosan supports the survival, migration, and differentiation of adult-derived neural stem and progenitor cells," Canadian Journal of Chemistry, vol. 88, no. 3, pp. 277-287, 2010.

[78] X. Lu, Z. Qiu, Y. Wan, Z. Hu, and Y. Zhao, "Preparation and characterization of conducting polycaprolactone/chitosan/ polypyrrole composites," Composites A, vol. 41, no. 10, pp. 1516-1523, 2010.

[79] Y. Wan, J. Gao, J. Zhang, W. Peng, and G. Qiu, "Biodegradability of conducting chitosan-g-polycaprolactone/polypyrrole conduits," Polymer Degradation and Stability, vol. 95, no. 10, pp. 1994-2002, 2010.

[80] C. Huang, R. Chen, Q. Ke, Y. Morsi, K. Zhang, and X. Mo, "Electrospun collagen-chitosan-TPU nanofibrous scaffolds for tissue engineered tubular grafts," Colloids and Surfaces B: Biointerfaces, vol. 82, no. 2, pp. 307-315, 2011.

[81] A. Cooper, N. Bhattarai, and M. Zhang, "Fabrication and cellular compatibility of aligned chitosan-PCL fibers for nerve tissue regeneration," Carbohydrate Polymers, vol. 85, no. 1, pp. 149-156, 2011.

[82] Y. C. Kuo and C. F. Yeh, "Effect of surface-modified collagen on the adhesion, biocompatibility and differentiation of bone marrow stromal cells in poly(lactide-co-glycolide)/chitosan scaffolds," Colloids and Surfaces B, vol. 82, no. 2, pp. 624-631, 2011.

[83] G. Ciapetti, L. Ambrosio, G. Marletta, N. Baldini, and A. Giunti, "Human bone marrow stromal cells: in vitro expansion and differentiation for bone engineering," Biomaterials, vol. 27, no. 36, pp. 6150-6160, 2006.
[84] R. A. Weber, W. C. Breidenbach, R. E. Brown, M. E. Jabaley, and D. P. Mass, "A randomized prospective study of polyglycolic acid conduits for digital nerve reconstruction in humans," Plastic and Reconstructive Surgery, vol. 106, no. 5, pp. 1036-1045, 2000.

[85] V. Hung and A. L. Dellon, "Reconstruction of a $4 \mathrm{~cm}$ human median nerve gap by including an autogenous nerve slice in a bioabsorbable nerve conduit: case report," Journal of Hand Surgery, vol. 33, no. 3, pp. 313-315, 2008.

[86] W. Fan, J. Gu, W. Hu et al., "Repairing a 35-MM-long median nerve defect with a chitosan/PGA artificial nerve graft in the human: a case study," Microsurgery, vol. 28, no. 4, pp. 238-242, 2008.

[87] X. Gu, F. Ding, Y. Yang, and J. Liu, "Construction of tissue engineered nerve grafts and their application in peripheral nerve regeneration," Progress in Neurobiology, vol. 93, no. 2, pp. 204-230, 2011. 


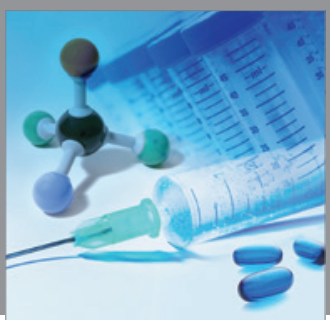

International Journal of

Medicinal Chemistry

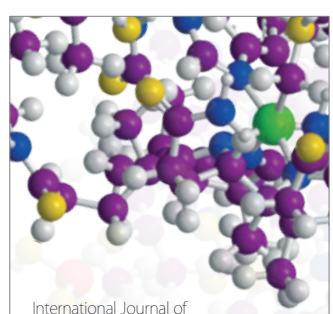

Carbohydrate Chemistry

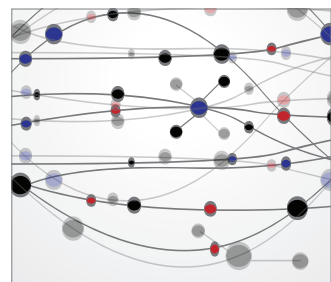

The Scientific World Journal
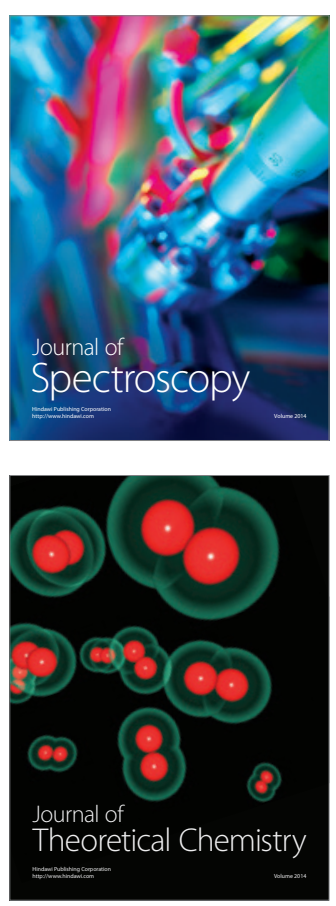
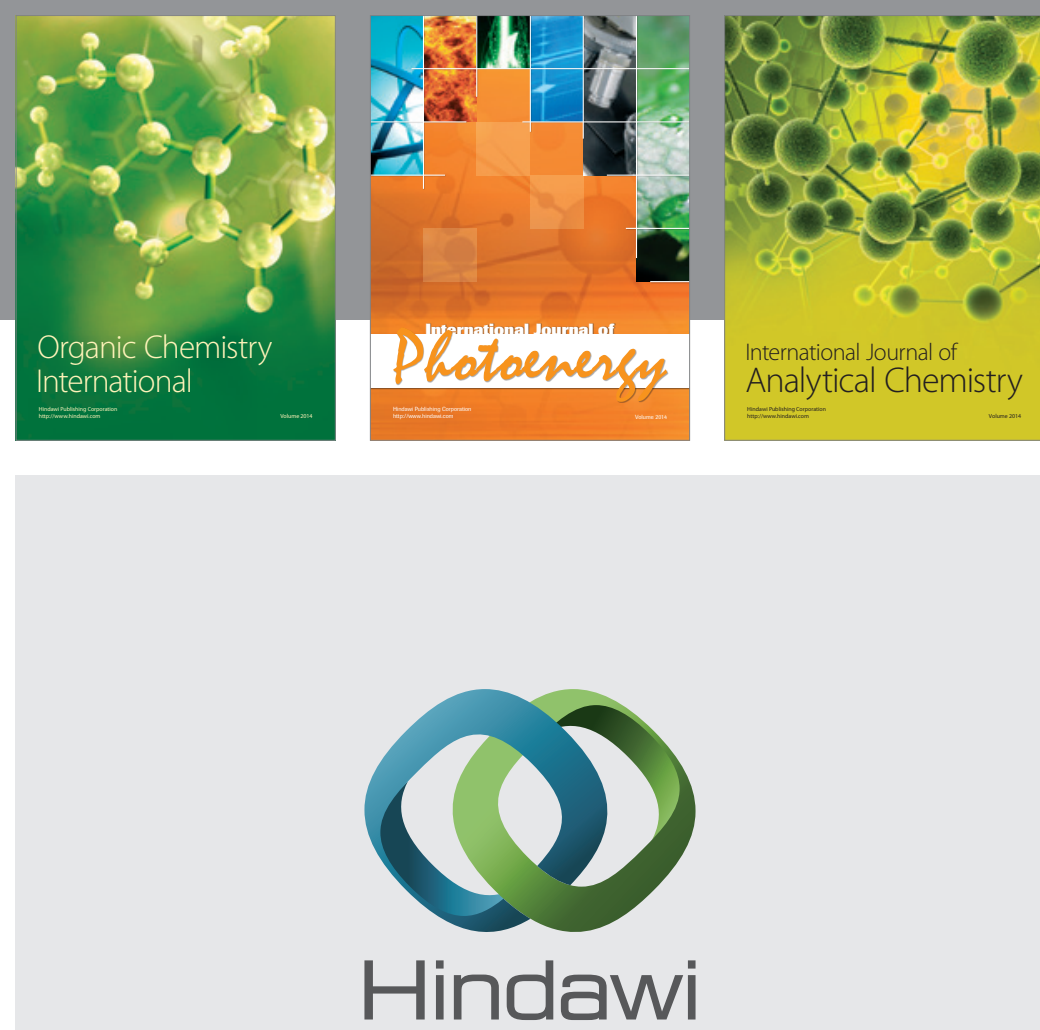

Submit your manuscripts at

http://www.hindawi.com
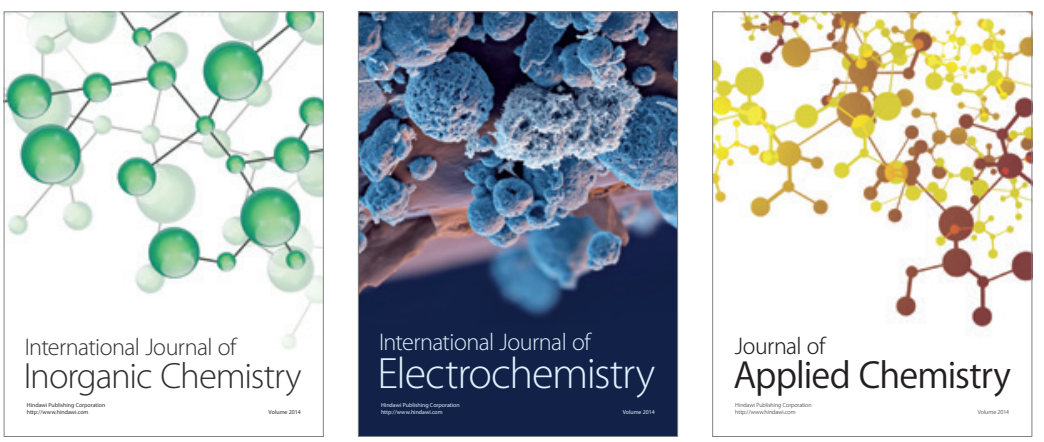

Journal of

Applied Chemistry
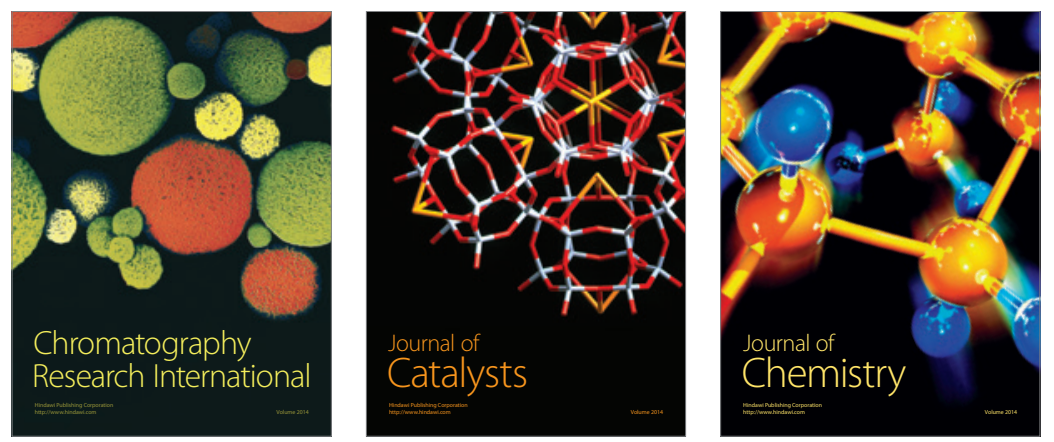
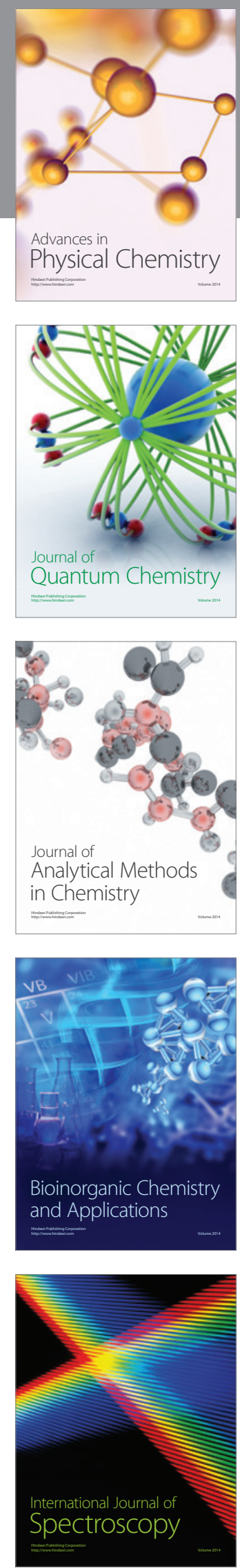